\title{
ERRATUM
}

\section{Review of photonic Hilbert transformers}

Chaotan SIMA (ه), James C. GATES, Michalis N. ZERVAS, Peter G. R. SMITH

Optoelectronics Research Centre, University of Southampton, Southampton SO17 1BJ, UK

(C) Higher Education Press and Springer-Verlag Berlin Heidelberg 2013

Front. Optoelectron. (2013) 6(1): 78-88

The author of Ref. [1] Frederick W K should be King F W.

The online version of the original article can be found at http://dx.doi.org/ 10.1007/s12200-012-0299-1 